\title{
Effect of physical exercise training in patients with Chagas heart disease: study protocol for a randomized controlled trial (PEACH study)
}

Fernanda de Souza Nogueira Sardinha Mendes ${ }^{1 *}$, Andréa Silvestre Sousa ${ }^{1}$, Fernando Cesar de Castro Cesar Souza², Vivian Liane Mattos Pinto ${ }^{1}$, Paula Simplicio Silva', Roberto Magalhães Saraiva ${ }^{1}$, Sergio Salles Xavier ${ }^{1}$, Henrique Horta Veloso', Marcelo Teixeira Holanda', Andréa Rodrigues Costa', Fernanda Martins Carneiro', Gilberto Marcelo Sperandio Silva', Juliana Pereira Borges ${ }^{2,3,4}$, Eduardo Tibirica ${ }^{2,4}$, Roberta Olmo Pinheiro ${ }^{4}$, Flávio Alves Lara ${ }^{4}$, Alejandro Marcel Hasslocher-Moreno ${ }^{1}$, Pedro Emmanuel Alvarenga Americano Brasil ${ }^{1}$ and Mauro Felippe Felix Mediano ${ }^{1}$

\begin{abstract}
Background: The effects of exercise training on Chagas heart disease are still unclear. This study aimed to evaluate the effect of exercise training over functional capacity, cardiac function, quality of life, and biomarkers in Chagas heart disease.

Methods: The PEACH study is a superiority randomized clinical trial which will include subjects who meet the following criteria: Chagas heart disease with a left ventricular ejection fraction below $45 \%$ with or without heart failure symptoms; clinical stability in the last 3 months; adherence to clinical treatment; and age above 18 years. The exclusion criteria are: pregnancy; neuromuscular limitations; smoking; evidence of non-chagasic heart disease; systemic conditions that limit exercise practice or cardiopulmonary exercise test; unavailability to attend the center three times a week during the intervention period; and practitioners of regular exercise. The intervention group will perform an exercise training intervention three times per week during 6 months and will be compared to the control group without exercise. Both groups will undergo the same monthly pharmaceutical and nutritional counseling as well as standard medical treatment according to the Brazilian consensus on Chagas disease. The primary outcome is functional capacity based on peak exercise oxygen consumption during cardiopulmonary exercise testing. Secondary outcomes are: cardiac function; body composition; muscle respiratory strength; microvascular reactivity; cardiac rhythm abnormalities; autonomic function; biochemical; oxidative stress and inflammatory biomarkers; and quality of life. Subjects will be evaluated at baseline, and at 3 and 6 months after randomization. Thirty patients will be randomly assigned into exercise or control groups at a ratio of 1:1.
\end{abstract}

Discussion: Findings of the present study will be useful to determine if physical exercise programs should be included as an important additional therapy in the treatment of patients with Chagas heart disease.

(Continued on next page)

\footnotetext{
* Correspondence: fernanda.sardinha@ini.fiocruz.br

${ }^{1}$ Evandro Chagas National Institute of Infectious Diseases, Oswaldo Cruz Foundation, Avenida Brasil 4365, Manguinhos, Rio de Janeiro 21040-360, Brazil

Full list of author information is available at the end of the article
} 
(Continued from previous page)

Trial registration: ClinicalTrials.gov ID: NCT02517632 (registered on 6 August 2015).

Keywords: Chagas heart disease, Heart failure, Exercise training, Cardiac rehabilitation, Cardiopulmonary exercise test

Abbreviations: A, Late diastolic filling velocity; APU, Arbitrary perfusion units; BMI, Body mass index; BPmax, Maximum achieved blood pressure; CHD, Chagas heart disease; CP, Circulatory power; CPET, Cardiopulmonary exercise test; CR, Cardiac rehabilitation; CV, Coefficient of variation; CVC, Cutaneous vascular conductance; E, Early diastolic filling velocity; E', Peak early diastolic myocardial velocity; EF, Ejection fraction; ELISA, Enzyme-linked immunosorbent assay; FAl, Functional aerobic impairment; HDL, High-density lipoprotein; HF, Heart failure; HFP, High-frequency power; HRmax, Maximum achieved heart hate; HRV, Heart rate variability; IFN- $\gamma$, Interferon gamma; IL-10, Interleukin 10; IL-1ß, Interleukin beta 1; IL-4, Interleukin 4; IL-8, Interleukin 8; INI, Evandro Chagas National Institute of Infectious Diseases; IPAQ-SF, International Physical Activity Questionnaire-Short Form; LDL, Low-density lipoprotein; LFP, Low-frequency power; LMM, Linear mixed models; LV, Left ventricular; LVEF, Left ventricular ejection fraction; MCP-1, Monocyte chemotactic protein 1; MEP, Maximal expiratory pressure; MIP, Maximal inspiratory pressure; NT-proBNP, N-terminal of the prohormone brain natriuretic peptide; $\mathrm{O}_{2}$ pulse, Oxygen pulse; OUES, Oxygen uptake efficiency slope; PEACH study, Exercise program in Chagas heart disease; PNN >50, Percentage of differences between adjacent normal-to-normal RR intervals that are greater than 50 ms; PORH, Post-occlusive reactive hyperemia; rMSSD, Root mean square of successive differences; RV, Right ventricular; RVS, Right ventricular peak systolic myocardial velocity; S, Peak systolic myocardial velocity; SDANN, Standard deviation of the averages of normal-to-normal RR intervals; SDNN, Standard deviation of normal-to-normal RR intervals; SDNNIDX, Mean of the standard deviations of normal-to-normal RR intervals; slope VE/ $\mathrm{VCO}_{2}$, Ventilation slope equivalent to carbon dioxide production; TAPSE, Tricuspid annular plane systolic excursion; TNF, Tumor necrosis factor; TP, Total power; VA, Presence of complex ventricular arrhythmias; $\mathrm{VCO}_{2} \mathrm{NO}_{2}$, Respiratory exchange ratio; VLDL, Very low-density lipoprotein; VLFP, Very low-frequency power; $\mathrm{VO}_{2}$ AT, Oxygen consumption at anaerobic threshold; $\mathrm{VO}_{2}$ peak, Oxygen consumption at peak of exercise

\section{Background}

Chagas heart disease (CHD) is the most common manifestation of chronic Chagas disease with prevalence of 20 to $30 \%$ in patients infected with Trypanosoma cruzi [1]. Patients with CHD present a high incidence of cardiac complications, morbidity, and mortality in Latin America [2]. The Brazilian consensus on Chagas disease [3] classifies CHD into different stages that reflect prognosis: stage A (abnormalities on electrocardiogram attributable to Chagas disease and no left ventricular (LV) wall motion abnormalities detected by echocardiography), stage B1 (LV wall motion abnormalities with an ejection fraction (EF) $>45 \%$ and no heart failure (HF)), stage B2 (LVEF $<45 \%$ and no HF), stage C (compensated HF), and stage D (endstage HF).

Currently, CHD therapy is based on treating symptoms and slowing the heart disease progression following the standard guidelines for cardiac disease of other etiologies [4]. However, some particular features in the clinical course of CHD demand specific treatment. Therefore, studies evaluating the effects of different strategies on patients with CHD are necessary.

Treatment of heart disease requires a multidisciplinary team-based care approach that includes exercise training to improve patients' functional status [5]. Cardiac rehabilitation (CR) is associated with consistent improvements in symptoms, cardiac mortality, number of hospitalizations, quality of life, and in numerous relevant clinical endpoints [6]. Moreover, exercise programs have gained increased recognition during the past years and have been strongly recommended by many different cardiology societies in the world, mainly for non-CHD [7-9].

However, exercise studies including patients with Chagas disease are warranted since these patients are usually not included in exercise clinical trials [10]. The first study that addressed the effects of exercise training on CHD showed that functional capacity, clinical symptoms, and some domains of health-related quality of life (vitality, emotional aspects, and mental health) improved after 3 months of follow-up [11]. Another single-arm study demonstrated that oxygen consumption at peak of exercise $\left(\mathrm{VO}_{2}\right.$ peak), oxygen pulse $\left(\mathrm{O}_{2}\right.$ pulse $)$, and oxygen consumption at anaerobic threshold $\left(\mathrm{VO}_{2} \mathrm{AT}\right) \mathrm{im}$ proved after 6 months of exercise training [12]. However, the interpretation of these results is limited by the short-term follow-up in the former study and the lack of a control group in the later study. Thus, new well-designed clinical trials are necessary to improve the knowledge of CR effects on patients with CHD.

The PEACH study, which stands for "Exercise Program in Chagas Heart Disease" in Portuguese, is designed to address the effects of exercise training in patients with CHD. The primary aim of the study is to investigate the effects of exercise on functional capacity 
in patients with $\mathrm{CHD}$, measured as the $\mathrm{VO}_{2}$ peak during a maximal progressive cardiopulmonary exercise test (CPET). Secondary objectives are to assess the effects of exercise training on cardiac function, body composition, muscle respiratory strength, microvascular reactivity, cardiac rhythm abnormalities, autonomic function, biochemical, inflammatory and oxidative stress biomarkers, and quality of life. We hypothesized that exercise training will be safe and will promote important clinical benefits mainly on functional capacity among patients with CHD.

\section{Methods}

\section{Study design}

The PEACH study is a single-center, superiority randomized clinical trial (ClinicalTrials.gov ID: NCT02517632) performed at the Evandro Chagas National Institute of Infectious Diseases (INI), a national reference center for treatment and research in infectious and tropical diseases in Rio de Janeiro, Brazil. The unit staff is composed of infectious disease specialists, cardiologists, gastroenterologists, nurses, pharmacists, and exercise physiologists. Resources such as echocardiography, computed tomography, digestive endoscopy, 24-h Holter electrocardiogram monitoring, ambulatory blood pressure monitoring, CPET, and cardiac rehabilitation are also available. The institute has outpatient and inpatient treatment sectors with an intensive care unit.

\section{Participants and recruitment}

Individuals followed at INI will be sequentially recruited to participate in the study. The eligibility criteria are: (1) Chagas disease diagnosis, confirmed by two simultaneous serological tests (enzyme-linked immunosorbent assay (ELISA) and indirect immunofluorescence) [3], (2) CHD with LVEF $<45 \%$ with or without HF symptoms at baseline evaluation (CHD stages B2 and C), (3) New York Heart Association class I or II in the previous 3 months, with clinical stability by investigator judgment, (4) clinical treatment according to HF guidelines, including treatment with angiotensin-converting enzyme inhibitors or angiotensin receptor blockers, and betablocker therapy, or documented rationale for variation, including intolerance, contraindication, or patient preference $[13,14]$. Patients will be on stable doses of medications for 6 weeks prior to enrollment, and (5) age above 18 years. Exclusion criteria are: (1) pregnancy, (2) neuromuscular limitations that preclude physical exercise, (3) smoking, (4) evidence of known non-chagasic heart disease, (5) systemic conditions that limit exercise practice or CPET, (6) unavailability to attend the center three times a week during the intervention period, and (7) practitioners of regular exercise.
Trained research assistants or study investigators will conduct the evaluations in a quiet and private room. Initial interviews will include a questionnaire to collect sociodemographic data (income, race, age, sex, and schooling), medical history (stage of CHD, comorbidities, medication, cardiac devices, and arrhythmias), functional class, and level of physical activity through the International Physical Activity Questionnaire-Short Form (IPAQ-SF). Evaluations of functional capacity (maximal progressive CPET), cardiac function (two-dimensional echocardiography), body composition (anthropometry and skinfolds), muscle respiratory strength (manovacuometry), microvascular reactivity (laser speckle flowmetry), cardiac rhythm abnormalities (24-h Holter), autonomic function (active orthostatic stress test), laboratorial biomarkers (biochemical, inflammatory, and oxidative stress), and quality of life (Short Form-36 (SF36) and Minnesota Living with Heart Failure Questionnaire) will be performed at baseline, after 3 months, and at the end of follow-up (6 months). After initial evaluation, patients will be randomized to intervention or control groups as seen in Fig. 1.

\section{Intervention}

Patients included in the intervention arm will be submitted to an exercise training performed three times a week for $60 \mathrm{~min}$, during a 6-month period. Each session will comprise $30 \mathrm{~min}$ of aerobic exercise on a treadmill or cycle ergometer (the first $5 \mathrm{~min}$ of warm-up and the last $5 \mathrm{~min}$ of cool-down), $20 \mathrm{~min}$ of strength exercises for the major muscle groups (sit-ups, push-ups, and pullups), and $10 \mathrm{~min}$ of stretching. The exercise intensity will be set according to the heart rate obtained during the CPET, corresponding to the anaerobic threshold minus $10 \%$ in the first month of exercise protocol and the anaerobic threshold plus $10 \%$ in the following months. Blood pressure, heart rate, and oxygen saturation will be measured before, during aerobic exercise (at $20 \mathrm{~min}$ of aerobic exercise), and at the end of each training session using an aneroid sphygmomanometer (Unitec, São Paulo, Brazil), a heart rate monitor (Polar FT1, Kempele, Finland), and an oximeter (IMFtec ${ }^{\circ}$, São Paulo, Brazil). Individuals with long QT syndrome, atrial fibrillation or flutter with ventricular response less than $100 \mathrm{bpm}$ at rest, frequent premature ventricular contractions, sinus tachycardia, sinus bradycardia, or patients with any cardiac related-symptoms will have their rhythm observed with a cardiac monitor during the exercise sessions. Glucose monitoring will be performed before and after exercise sessions in diabetic patients to guarantee its safety. For pre-exercise blood glucose levels of less than $100 \mathrm{mg} . \mathrm{dL}^{-1}\left(5.5 \mathrm{mmol} . \mathrm{L}^{-1}\right)$, the American Diabetes Association recommends that carbohydrate should be ingested before any exercise. The patient will be able to engage in exercise with levels exceeding 


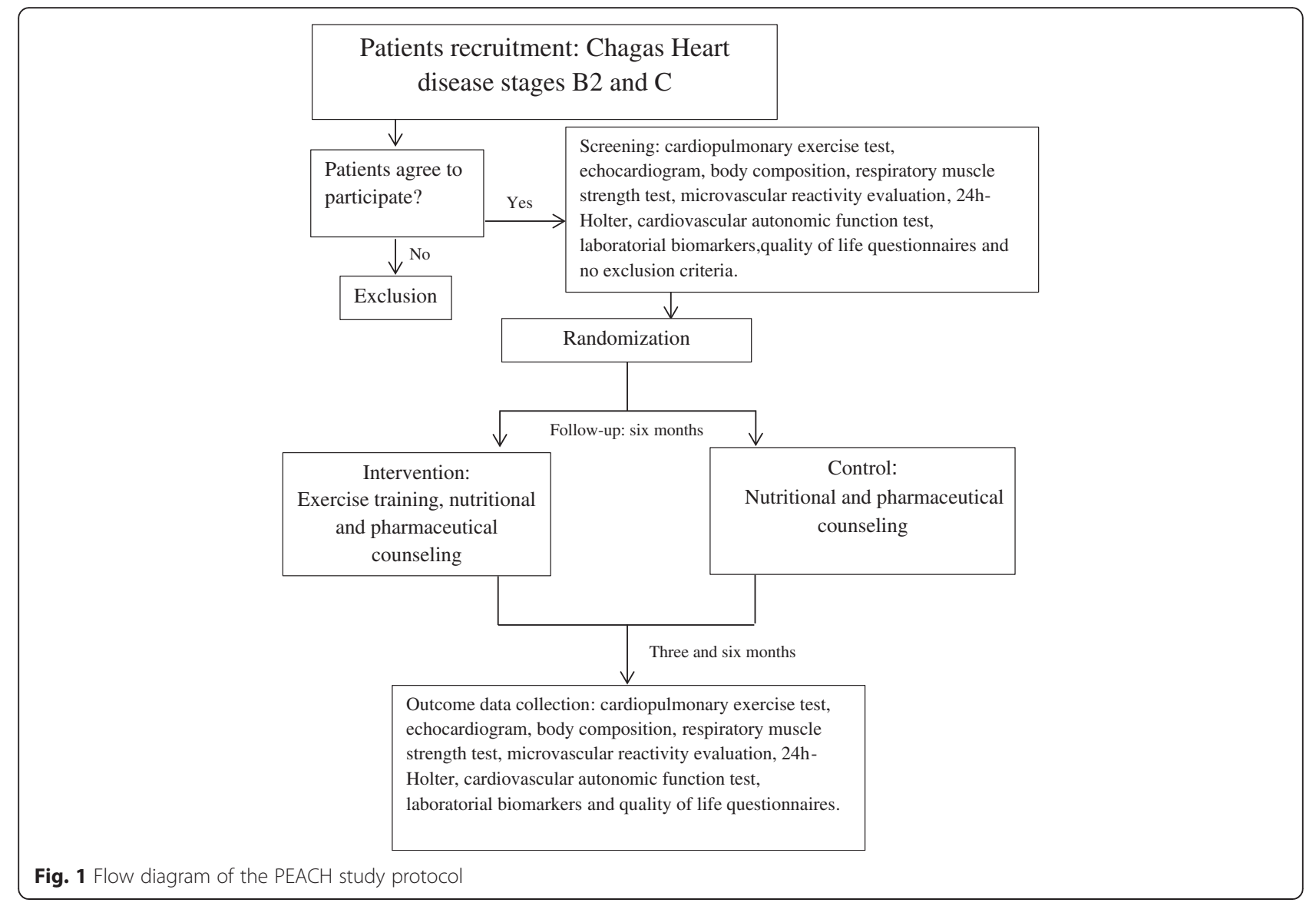

$300 \mathrm{mg} \cdot \mathrm{dL}^{-1}\left(16.7 \mathrm{mmol} . \mathrm{L}^{-1}\right)$ unless they present with ketosis symptoms, or dehydration [15]. All training sessions will be performed in the morning, indoors, and with a controlled temperature environment under supervision of medical staff.

Patients of both groups will undergo regular medical appointments with the same cardiologist during the follow-up based on standard medical treatment of the Brazilian consensus on Chagas disease recommendations [3] and with other medical specialties if necessary. In addition, nutritional and pharmaceutical counseling will be provided monthly for both groups. The nutritional counseling will consist of general guidance about healthy eating habits and will include how to reduce saturated fat and include poly and monounsaturated fatty acids, to consume more vitamins, high-fiber carbohydrates, and to reduce the sodium and water intake for patients with $\operatorname{HF}[16,17]$. Pharmacists will guide patients about medication usage, drug dosage, and compliance. In order to reduce any bias due to medication noncompliance, all patients will receive monthly personalized packages according to the medical prescription. The pills will be organized by the time and days that the pills should be taken (Fig. 2). The schedule of enrollment, interventions, and assessments of the PEACH study is seen in Fig. 3 and the Standard Protocol Items: Recommendations for Interventional Trials (SPIRIT) checklist in Additional file 1.

\section{Outcomes}

These will be functional capacity, cardiac function, body composition, muscle respiratory strength, microvascular reactivity, cardiac rhythm abnormalities, autonomic function, biochemical, oxidative stress and inflammatory biomarkers, and quality of life will be assessed at baseline, 3 months, and 6 months of the study.

\section{Cardiopulmonary exercise test}

The primary outcome is functional-capacity-based on $\mathrm{VO}_{2}$ peak obtained by a CPET using the $\mathrm{VO}_{2000}$ gas analyzer (MedGraphics ${ }^{\circ}$, St. Paul, MS, USA) with a computerized system Ergo PC Elite (Micromed ${ }^{\circ}$, Brasília, Brazil) and a treadmill (Inbramed ${ }^{\circ}$, Porto Alegre, Brazil). A blinded evaluator will perform an incremental exercise test using a ramp protocol, tailored to achieve a fatigue limited exercise duration of approximately 8 to $12 \mathrm{mi}$ nutes. [18]. The workloads will be based on age, gender, 


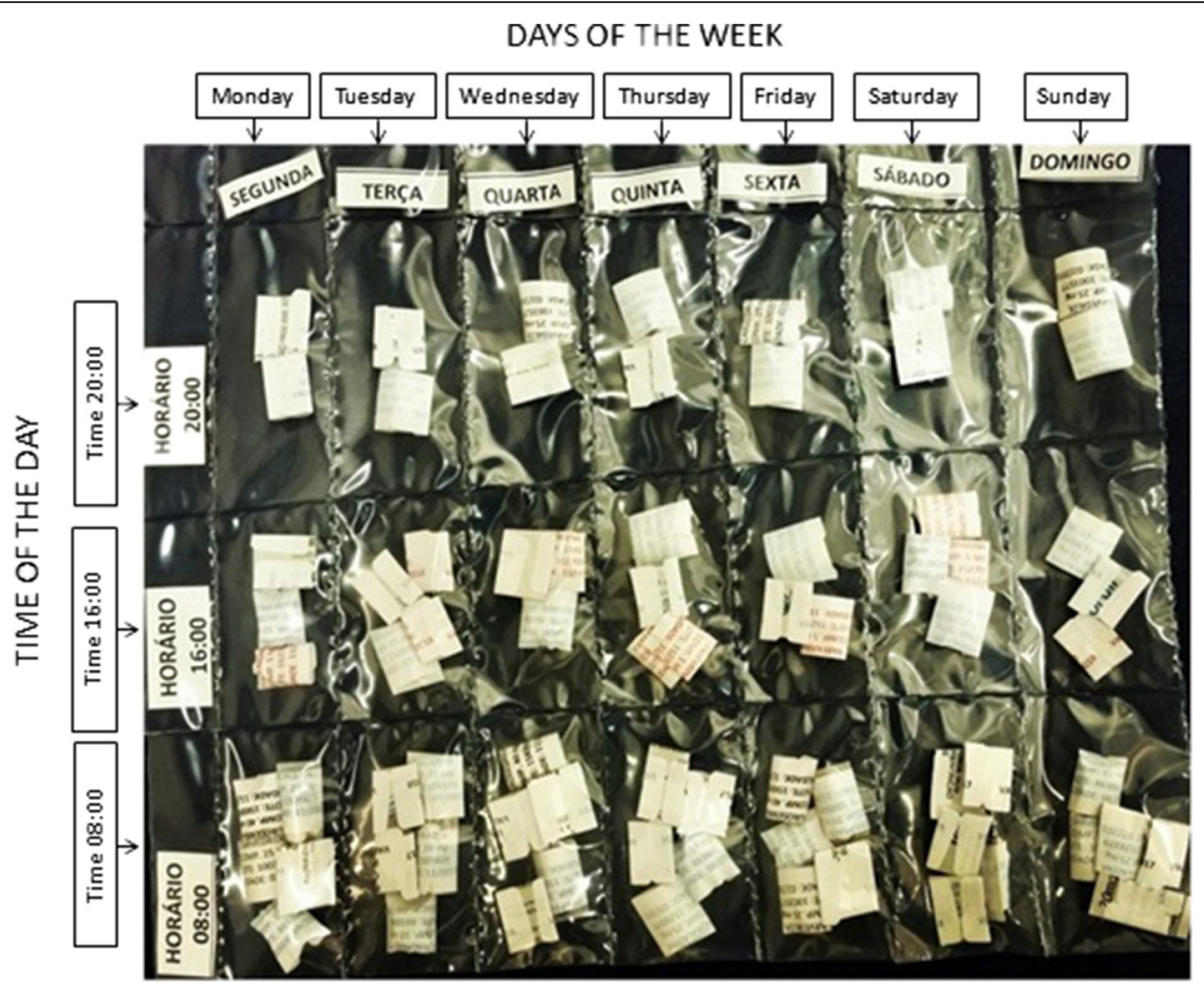

Fig. 2 Medication packages

height, and weight; adapted to each subject's physical condition and effort tolerance. Gas and volume calibrations will be executed on the early morning of each test day. Pulmonary gas exchange will be analyzed breathby-breath and averaged every $10 \mathrm{~s}$. A 12-lead electrocardiogram will monitor heart rhythm during CPET.

The CPET will be limited by symptoms through the subjective fatigue perception scale (Borg modified) ranging from 0 to 10 , with 0 representing absence of fatigue and 10 maximum tolerated efforts. The examiner may interrupt the test in case of identification of any harmful hemodynamic response. The recovery phase will be active with walking in a pre-determined velocity of $2 \mathrm{~km} \cdot \mathrm{h}^{-1}$ and $2 \%$ of inclination.

The following CPET variables will be analyzed: maximum achieved heart hate (HRmax); maximum achieved blood pressure (BPmax); respiratory exchange ratio $\left(\mathrm{VCO}_{2} / \mathrm{VO}_{2}\right)$; oxygen consumption at peak of exercise $\left(\mathrm{VO}_{2}\right.$ peak); oxygen consumption at anaerobic threshold $\left(\mathrm{VO}_{2} \mathrm{AT}\right)$; oxygen pulse $\left(\mathrm{O}_{2}\right.$ pulse); ventilation slope equivalent to carbon dioxide production (slope $\mathrm{VE} /$ $\mathrm{VCO}_{2}$ ); circulatory power $(\mathrm{CP})$; presence of complex ventricular arrhythmias (VA); functional aerobic impairment (FAI); and oxygen uptake efficiency slope (OUES) values.

The $\mathrm{VO}_{2}$ peak during exercise will be defined as the greatest value during $30 \mathrm{~s}$ before and after maximum effort. The $\mathrm{VO}_{2}$ AT will be determined by the point at which expired carbon dioxide increases in a nonlinear fashion relative to the rate of oxygen consumption according to the V-slope method. Ergo PC Elite software will determine the other variables obtained on the CPET.

Before CPET, the rest electrocardiogram will be evaluated to define if the cardiac rhythm is acceptable to perform the exam. The following markers will not be accepted: sustained ventricular tachycardia, second- and third-degree atrioventricular block, atrial or supraventricular tachycardia (more than $100 \mathrm{bpm}$ ).

\section{Cardiac function}

Patients will undergo a standard two-dimensional resting echocardiogram using a phased-array ultrasound system (Vivid 7, General Electric Medical Systems ${ }^{\circ}$, Milwaukee, WI, USA) equipped with a M4S transducer. Cardiac dimensions, LV and right ventricular (RV) systolic function, and Doppler measurements will be measured as recommended by the American Society of Echocardiography [19-21]. The biplane Simpson's method will be used to estimate LVEF. The other studied variables will be as follows: RV peak systolic myocardial velocity (RVS), tricuspid annular plane systolic excursion (TAPSE), RV systolic pressure, peak early (E) and late 


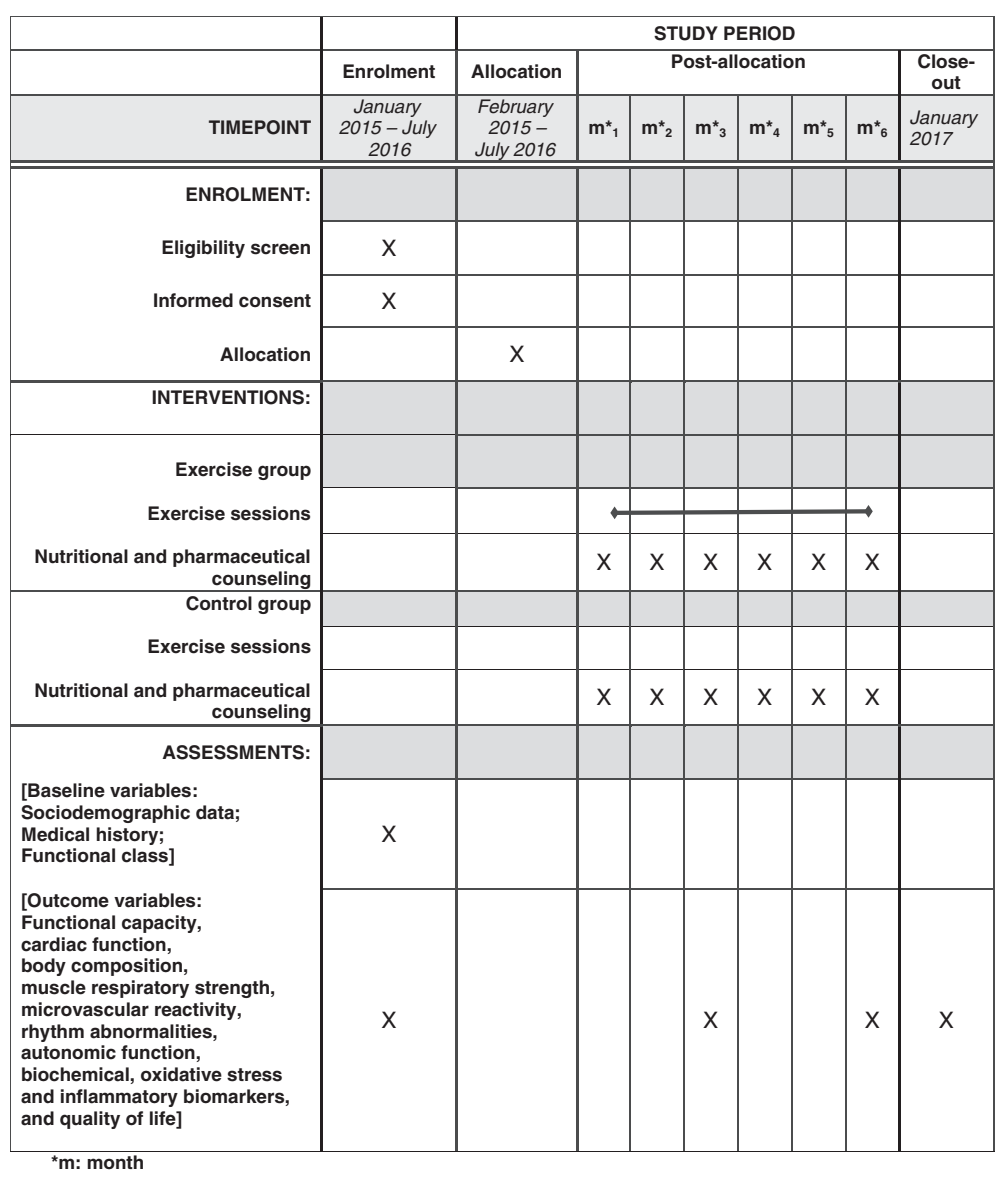

Fig. 3 Schedule of enrollment, interventions, and assessments of the PEACH study

(A) diastolic filling velocities, E/A ratio, peak, and peak early (E') diastolic myocardial velocities.

\section{Body composition}

The anthropometric evaluation will consist of measurements of body weight and height with minimal clothing and without shoes using a calibrated digital scale with coupled stadiometer. A ratio between weight $(\mathrm{kg})$ and squared height $(\mathrm{m})$ will determine body mass index (BMI), an important surrogate of nutritional status [22]. Two circumferences will be taken: the waist circumference at the narrowest waist level and the hip circumference at the largest circumference around the buttocks [23].

A seven-site skinfold thickness protocol including chest, midaxillary, triceps, subscapular, abdomen, suprailiac, and thigh sites will be used to evaluate body composition. Measurements will be taken twice on the right side of the body while standing in a relaxed position (Lange skinfold caliper, Beta Technology Inc., Cambridge, MD, USA). The average of each of these seven skinfold thickness will be summated and used to estimate body composition using the Jackson and Pollock equation $[24,25]$.

\section{Respiratory muscle strength}

Respiratory muscle strength will be assessed by maximal inspiratory (MIP) and expiratory pressures (MEP) using a digital pressure manometer connected to a mouthpiece (MVD $3000^{\circ}$, Globalmed, Brazil). Patients will remain in a seated position with a nose clip. They will be requested to make a maximum inspiratory effort at residual volume and a maximum expiratory effort at total lung capacity, sustaining it for 1 to $2 \mathrm{~s}$ [26]. Once the operator is satisfied, the maximum value of three maneuvers that vary by less than $20 \%$ will be recorded [27].

\section{Microvascular reactivity}

A laser speckle contrast imaging system with a laser wavelength of $785 \mathrm{~nm}$ (PeriCam PSI system, Perimed, Järfälla, Sweden), coupled to iontophoresis of acetylcholine and sodium nitroprusside, will noninvasively measure real time cutaneous microvascular flow changes in the forearm $[28,29]$. 
For the post-occlusive reactive hyperemia (PORH) test, arterial occlusion will be performed with suprasystolic pressure (50 $\mathrm{mmHg}$ above the systolic arterial pressure) using a sphygmomanometer applied to the arm of the subject over 3 min. Peak skin flow will be measured after pressure release.

Images will be analyzed using the manufacturer's software (PIMSoft, Perimed, Järfälla, Sweden). The measurements of skin blood flow will be divided by the mean arterial pressure to yield the cutaneous vascular conductance (CVC) in arbitrary perfusion units (APU) $/ \mathrm{mmHg}$, to avoid interference of blood pressure levels on microvascular flow.

\section{Cardiac rhythm abnormalities}

Arrhythmias and heart rate variability (HRV) will be evaluated with a 24-h Holter (portable three-channel recorder and analyzer; Cardio Light ${ }^{\oplus}$ and CardioSmart ${ }^{\oplus}$ 5.0, Cardio System, São Paulo, Brazil). Patients will be requested to maintain their normal daily activities during the exam. Standard frequency- and time-domain heart rate variability indexes will be measured and evaluated in patients who do not have artificial pacing or atrial fibrillation rhythm. Only tracings of at least $18 \mathrm{~h}$ will be studied. Standard time-domain HRV indices (SDNN: standard deviation of normal-to-normal RR intervals; SDANN: standard deviation of the averages of normalto-normal RR intervals in all 5-min segments of a 24-h recording; SDNNIDX: mean of the standard deviations of normal-to-normal RR intervals in all 5-min segments of a 24-h recording; rMSSD: root mean square of successive differences; and $\mathrm{PNN}>50$ : percentage of differences between adjacent normal-to-normal RR intervals that are greater than $50 \mathrm{~ms}$ ) and frequency domain (TP: total power; VLFP: very low-frequency power; LFP: low-frequency power; HFP: high-frequency power) will be calculated [30-32].

\section{Cardiovascular autonomic function test}

The active orthostatic stress test consists of the evaluation of the heart rate and blood pressure response obtained from orthostatic change. The patient will rest for $5 \mathrm{~min}$ in the supine position, which will be followed by a quick stand-up position (3 to $5 \mathrm{~s}$ ). The electrocardiogram will be digitally recorded (ErgoMet 13 V1.0.3.0 HW Heart Ware, Porto Alegre, Brazil) $10 \mathrm{~s}$ before the maneuver and will last until $40 \mathrm{~s}$ after. The RR intervals will be measured throughout the test period. The baseline average heart rate will be based on the $10 \mathrm{RR}$ intervals immediately preceding the maneuver. The maximum $R R$ at rest over the minimum $R R$ after standup ratio will be calculated (index max:min RR) [33]. The blood pressure will be measured after $5 \mathrm{~min}$ at rest and the systolic blood pressure at $5 \mathrm{~s}$ after standing with diastolic blood pressure collected within $5 \mathrm{~s}$ after recording systolic blood pressure immediately after standing up to evaluate postural hypotension. Patients with artificial pacing or atrial fibrillation rhythm during the exam will not be evaluated.

\section{Laboratorial biomarkers}

A laboratory accredited by the College of American Pathologists will perform biochemical measurements. Total cholesterol, high-density lipoprotein (HDL) cholesterol, triacylglycerol, glucose, glycated hemoglobin, and the $\mathrm{N}$-terminal of the prohormone brain natriuretic peptide (NT-proBNP) will be measured using Siemens Dimension $^{\odot}$ reagent cartridge with an intra- and inter-assay coefficient of variation $(\mathrm{CV})<5 \%$. The Friedewald equation, based on the triacylglycerol measures, will be used to determine low-density lipoprotein (LDL) cholesterol and very low-density lipoprotein (VLDL) cholesterol concentrations [34].

Cytokine serum levels will be measured accordingly to the manufacturer's instructions (EBioscience, San Diego, CA, USA). Antibodies specific for interferon gamma (IFN- $\gamma)$, tumor necrosis factor (TNF), interleukin-beta 1 (IL-1 $\beta$ ), interleukin-10 (IL-10), interleukin-4 (IL-4), interleukin-8 (IL-8), or monocyte chemotactic protein 1 (MCP-1) will be coated onto the 96-well ELISA microplate overnight. Washing solution will be added to each well three times. Standards and unknown samples will be pipetted into these wells and will be incubated for 2 h. After washing, a biotinylated (detection) antibody specific for the described cytokines will be added and incubated for $1 \mathrm{~h}$. After washing, streptavidin-horseradish peroxidase will be added. After incubation for $30 \mathrm{~min}$ and washing to remove all unbound enzyme, color development solution will be added. Then, the plates will be read using a microplate reader (SpectraMax 190, Molecular Devices, Sunnyvale, CA, USA) at $450 \mathrm{~nm}$. Oxidative stress will be accessed by two different methodologies: detection of serum carbonylated proteins and reduced/oxidized glutathione ratio. The oxidative modified serum proteins will be detected after derivatization with 2,4-dinitrophenylhydrazine, through generation of dinitrophenylhydrazone, which will be analyzed in a spectrophotometer at $380 \mathrm{~nm}$ [35]. The reduced and oxidized glutathione pool will be determined in patients' sera using Detect $\mathrm{X}^{\oplus}$ Glutathione Fluorescent Detection Kit (Arbor Assays, Ann Arbor, MI, USA) as recommended by the manufacturer.

\section{Quality of life}

The short-form version of the SF-36 Health Survey [36] and the Minnesota Living with Heart Failure Questionnaire [37] will be used to assess quality of life, all of them previously translated and validated in Portuguese. 
The SF-36 consists of 36 questions in eight different domains: general health, physical functioning, social functioning, mental health, physical role, emotional role, bodily pain, and vitality. Each of these dimensions range from 0 (worst possible health state) to 100 (best possible health state).

The Minnesota Living with Heart Failure Questionnaire has 21 questions about how the heart disease influences the lifestyle related to physical, psychological, and social areas. Each question's responses range from 0 (none) to 5 (very much) and the maximum score is 105 . In this questionnaire, lower scores mean better quality of life.

\section{Sample size}

Considering a difference in peak oxygen intake of $2.9 \mathrm{ml} \cdot \mathrm{kg}^{-1} \cdot \mathrm{min}^{-1}$ with a standard deviation of $2.0 \mathrm{ml} . \mathrm{kg}^{-1} \cdot \mathrm{min}^{-1}$ [38], assuming an $\alpha=0.05$ and $\beta=0.20$, and increasing the sample size by $50 \%$ accounting for losses to follow-up, a total of 30 patients (15 in the control group and 15 in the exercise group) will be included.

\section{Randomization}

A sequence will be computer-generated to randomly allocate 30 patients into two groups in a 1:1 ratio (WinPepi version 11). The sequence will be generated in blocks and by strata of CHD classification (B2 and C) by a single researcher not involved in recruitment. Opaque envelopes will be filled in sequentially to either control or exercise group. Block size will be blinded from investigators involved in patients' recruitment.

\section{Blinding}

Given that exercise implies a behavioral intervention, it is not feasible to blind the patients. However, the evaluators will be blinded to the primary endpoint obtained by the CPET and the following secondary endpoints: microvascular reactivity, cardiac rhythm abnormalities, cardiovascular autonomic function test, and laboratorial biomarkers. A blinded researcher will perform all data analysis.

\section{Interim analysis and stopping rules}

Three interim analyses are planned. The first will be conducted when the tenth volunteer completes 3 months of follow-up, the second when the twentieth volunteer completes 3 months of follow-up and the third when the last volunteer completes 3 months of follow-up.

Trial interruption for ethical reasons due to either positive or negative results exceeding expectations may be recommended by an independent committee. The prespecified stopping rule is a difference of $50 \%$ in $\mathrm{VO}_{2}$ peak between groups, serious adverse events twice as frequent in one of the groups as cardiovascular death, acute myocardial infarction, unstable angina, cardiopulmonary arrest, malignant ventricular arrhythmias, decompensated
HF, and stroke. All these estimates should have a significance level of 0.01 or less in any of the interim analyses.

\section{Statistical analysis}

Descriptive analysis will consist of mean and standard deviation for continuous variables and percentage for categorical variables. Skewness and Kurtosis testing will be performed to assess the normality of data which will be log-transformed in case of skewed distribution. Variables that can change prognosis of the disease will be compared at baseline in relation to the exercise and control groups. Longitudinal effects of exercise on primary and secondary outcomes will be evaluated through linear mixed models (LMM), which correlate with repeated measures over the time. LMM is an intention-to-treat analysis as it includes all observations of each one of the patients regardless of losses to follow-up or noncompliance to exercise protocol. The longitudinal analysis will be made by the treatment $\times$ time interaction, which estimates the rate of changes in the outcomes. Residual plots of all models will be examined and the likelihoodratio test will be used to compare and select random intercept or random slope models.

The REDCap software will be used for data management and the data analysis will be conducted by Stata 13.0 software. Statistical significance will be set at $p<0.05$ for all analyses.

\section{Discussion}

Despite major advances in cardiovascular therapies, CHD still stands as an important cause of premature death in Latin America. Although the number of new cases of Chagas disease has decreased steadily since the late 1990s, many chronic cases are still part of routine care in public hospitals where patients with lower income have access to treatment. Moreover, decreased barriers to international travel and migration has led to an increase in migration of patients from Chagas disease-affected areas to nonendemic countries of North America and Europe. This globalization phenomenon transformed Chagas disease into a global medical challenge $[39,40]$.

CHD treatment is based on trials that studied the effect of different drugs on ventricular dysfunction, survival, and quality of life in patients with cardiomyopathies from other etiologies [4, 41]. However, CHD has a specific autonomic imbalance, a different pattern of myocardial fibrosis associated with an inflammatory milieu generated by parasite and host defenses, an increased risk of complex arrhythmias and a known worse prognosis than cardiomyopathies from other etiologies [42, 43].

Exercise training is becoming an important strategy in the treatment of patients with cardiac diseases. Numerous 
studies demonstrate that regular exercise is safe and associated with substantial benefits in patients with cardiovascular disease, mainly from ischemic etiology. Clinical adaptations to exercise training include improvements in functional capacity, enhancements in cardiac and vascular function, autonomic nervous system modulation, decreases in oxidative stress and low-grade inflammation, and improvements in lipid and glucose profiles [44, 45].

Despite these well-established benefits of exercise training in cardiac patients, there are few studies analyzing its effects on patients with CHD. Currently, only one randomized clinical trial [11] including 40 patients with CHD showed that exercise induced improvements in functional capacity and health-related quality of life. This study demonstrates that exercise is feasible, effective, and safe in patients with CHD but with restrictions as the indirect measurement of $\mathrm{VO}_{2}$ peak, the short-term follow-up and the inclusion of patients in the early stages of CHD, which preclude a definite conclusion about the effects of exercise training in this population.

In the PEACH study, we will try to fill this knowledge gap and address the issue of whether patients with $\mathrm{CHD}$ have the same benefits promoted by exercise training in patients with cardiomyopathies from other etiologies. We hypothesize that exercise training will be safe and promote improvements in functional capacity and quality of life, as previously demonstrated by Lima et al. [11] and Fialho et al. [12] in a different sample of patients with CHD. Since an exacerbated inflammatory response is an important mechanism involved in the development of CHD [46] and because several studies have been demonstrating an important anti-inflammatory property of exercise training [47], a decrease in the serum levels of pro-inflammatory cytokines and an increase in the serum levels of anti-inflammatory cytokines in patients with CHD in the exercise group is expected. Although enhancements in autonomic function as results of exercise training are present in studies with other cardiomyopathies [48], a recent article did not confirm this finding in patients with CHD [30]. We also hypothesize that cardiac function will improve by an increase in LVEF and an improvement in diastolic function, as seen in coronary artery disease [49], and that exercise will improve body composition, respiratory strength, microvascular reactivity, and oxidative stress agents based on results from studies evaluating exercise in non-CHD patients [50,51].

The benefits of exercise training that will be described by this study will set a new treatment strategy for CHD patients and that this strategy could be routinely included in clinical practice.

\section{Trial status}

Participants are currently being recruited.

\section{Additional file}

Additional file 1: SPIRIT checklist. (DOC $121 \mathrm{~kb}$ )

\section{Acknowledgements}

The authors wish to thank the Evandro Chagas National Institute of Infectious Diseases, the National Institute of Cardiology, and the Oswaldo Cruz Institute for clinical and laboratorial support.

\section{Funding}

This study was funded by the 6th Strategic Program for Support of Health from Oswaldo Cruz Foundation and National Council for Scientific and Technological Development (FIOCRUZ/CNPq). (No. 407742/2012-3).

\section{Availability of data and materials}

Not applicable.

\section{Authors' contributions}

FSNSM was involved in trial design, project development, manuscript drafting and review and will recruit, select, and collect clinical data from the patients. ASS was involved in trial design, manuscript drafting and review, acquisition of funding, and will collect clinical data from the patients. FCCS, RMS, HHV, ARC, JPB, EVT, ROP, FAL, AMHM, and PEAAB were involved in manuscript drafting or review and will collect clinical data from the patients. VLMP, PSS, SSX, MTH, and GMSS were involved in trial design and will collect clinical data from the patients. FMC will be involved in patient evaluations and will collect clinical data from the patients. MFFM was involved in trial design, manuscript drafting and review and will collect clinical data from the patients. All authors read and approved the final manuscript.

\section{Authors' information}

Not applicable.

\section{Competing interests}

The authors declare that they have no competing interests.

Consent for publication

Not applicable.

\section{Ethical approval and consent to participate}

The trial was approved by the Evandro Chagas National Institute of Infectious Diseases Research Ethics Committee (CAAE 38038914.6.0000.5262). All participants will read and sign a written informed consent and will be advised that they can decline to respond to any question or refuse to continue the research any time without compromising their treatment. All participants will receive travel allowance for the evaluations and exercise sessions provided by the project sponsor. Patients followed in the trial who sustain any harm from the intervention will be monitored and will have access to treatment until resolution of the clinical picture.

\section{Author details}

${ }^{1}$ Evandro Chagas National Institute of Infectious Diseases, Oswaldo Cruz Foundation, Avenida Brasil 4365, Manguinhos, Rio de Janeiro 21040-360, Brazil. ${ }^{2}$ National Institute of Cardiology, Rua das Laranjeiras 374, Laranjeiras, Rio de Janeiro 22240-006, Brazil. ${ }^{3}$ Physical Education and Sports Institute, State University of Rio de Janeiro, Rua São Francisco Xavier, 524, Maracanã, Rio de Janeiro 20550-900, Brazil. ${ }^{4}$ Oswaldo Cruz Institute, Oswaldo Cruz Foundation, Avenida Brasil 4365, Manguinhos, Pavilhão Cardoso Fontes, Sala 64, Rio de Janeiro 21040-360, Brazil.

Received: 21 April 2016 Accepted: 13 August 2016

Published online: 02 September 2016

References

1. Bern C. Chagas' disease. N Engl J Med. 2015;373(5):456-66.

2. Rassi Jr A, Rassi A, Marin-Neto JA. Chagas disease. Lancet. 2010;375(9723):1388-402.

3. Ministerio da Saude. Secretaria de Vigilancia em S. Brazilian consensus on Chagas disease. Rev Soc Bras Med Trop. 2005;38 Suppl 3:7-29. 
4. Ribeiro AL, Nunes MP, Teixeira MM, Rocha MO. Diagnosis and management of Chagas disease and cardiomyopathy. Nat Rev Cardiol. 2012;9(10):576-89.

5. Brush Jr JE, Handberg EM, Biga C, Birtcher KK, Bove AA, Casale PN, Clark MG, Garson Jr A, Hines JL, Linderbaum JA, et al. 2015 ACC Health Policy Statement on Cardiovascular Team-Based Care and the Role of Advanced Practice Providers. J Am Coll Cardiol. 2015;65(19):2118-36.

6. Ades PA, Keteyian SJ, Balady GJ, Houston-Miller N, Kitzman DW, Mancini DM, Rich MW. Cardiac rehabilitation exercise and self-care for chronic heart failure. JACC Heart Fail. 2013;1(6):540-7.

7. McMurray JJ, Adamopoulos S, Anker SD, Auricchio A, Bohm M, Dickstein K, Falk V, Filippatos G, Fonseca C, Gomez-Sanchez MA, et al. ESC guidelines for the diagnosis and treatment of acute and chronic heart failure 2012: The Task Force for the Diagnosis and Treatment of Acute and Chronic Heart Failure 2012 of the European Society of Cardiology. Developed in collaboration with the Heart Failure Association (HFA) of the ESC. Eur J Heart Fail. 2012;14(8):803-69.

8. European Association of Cardiovascular Prevention, Rehabilitation Committee for Science G, Eacpr, Corra U, Piepoli MF, Carre F, Heuschmann P, Hoffmann U, Verschuren M, Halcox J, et al. Secondary prevention through cardiac rehabilitation: physical activity counselling and exercise training: key components of the position paper from the Cardiac Rehabilitation Section of the European Association of Cardiovascular Prevention and Rehabilitation. Eur Heart J. 2010;31(16):1967-74.

9. Pina IL, Apstein CS, Balady GJ, Belardinelli R, Chaitman BR, Duscha BD, Fletcher BJ, Fleg JL, Myers JN, Sullivan MJ, et al. Exercise and heart failure: a statement from the American Heart Association Committee on exercise, rehabilitation, and prevention. Circulation. 2003;107(8):1210-25.

10. Bocchi EA. Exercise training in Chagas' cardiomyopathy: trials are welcome for this neglected heart disease. Eur J Heart Fail. 2010;12(8):782-4.

11. Lima MM, Rocha MO, Nunes MC, Sousa L, Costa HS, Alencar MC, Britto RR, Ribeiro AL. A randomized trial of the effects of exercise training in Chagas cardiomyopathy. Eur J Heart Fail. 2010;12(8):866-73.

12. Fialho PH, Tura BR, Sousa AS, Oliveira CR, Soares CC, Oliveira JR, Souza MV, Coelho MP, Souza FC, Cunha AB, et al. Effects of an exercise program on the functional capacity of patients with chronic Chagas' heart disease, evaluated by cardiopulmonary testing. Rev Soc Bras Med Trop. 2012;45(2):220-4.

13. Yancy CW, Jessup M, Bozkurt B, Butler J, Casey DE, Drazner MH, Fonarow GC, Geraci SA, Horwich T, Januzzi JL, et al. 2013 ACCF/AHA guideline for the management of heart failure: a report of the American College of Cardiology Foundation/American Heart Association Task Force on practice guidelines. Circulation. 2013;128(16):e240-327.

14. Ponikowski P, Voors AA, Anker SD, Bueno H, Cleland JG, Coats AJ, Falk V, González-Juanatey JR, Harjola VP, Jankowska EA, et al. 2016 ESC Guidelines for the diagnosis and treatment of acute and chronic heart failure: The Task Force for the diagnosis and treatment of acute and chronic heart failure of the European Society of Cardiology (ESC). Developed with the special contribution of the Heart Failure Association (HFA) of the ESC. Eur J Heart Fail. 2016;18(8): 891-975.

15. Colberg SR, Albright AL, Blissmer BJ, Braun B, Chasan-Taber L, Fernhall B, Regensteiner JG, Rubin RR, Sigal RJ, Medicine ACoS, et al. Exercise and type 2 diabetes: American College of Sports Medicine and the American Diabetes Association: joint position statement. Exercise and type 2 diabetes. Med Sci Sports Exerc. 2010;42(12):2282-303.

16. daSaúde M. Dietary guidelines for the Brazilian population. 2nd ed. Brasília: Ministry of Health of Brazil; 2014.

17. Santos RD, Gagliardi AC, Xavier HT, Magnoni CD, Cassani R, Lottenberg AM, De Sociedade Brasileira C, Arpadi Faludi A, Geloneze B, Scherr C, et al. First guidelines on fat consumption and cardiovascular health. Arq Bras Cardiol. 2013;100(1 Suppl 3):1-40.

18. Balady GJ, Arena R, Sietsema K, Myers J, Coke L, Fletcher GF, Forman D, Franklin B, Guazzi M, Gulati M, et al. Clinician's guide to cardiopulmonary exercise testing in adults: a scientific statement from the American Heart Association. Circulation. 2010;122(2):191-225.

19. Lang RM, Badano LP, Mor-Avi V, Afilalo J, Armstrong A, Ernande L, Flachskampf FA, Foster E, Goldstein SA, Kuznetsova T, et al. Recommendations for cardiac chamber quantification by echocardiography in adults: an update from the American Society of Echocardiography and the European Association of Cardiovascular Imaging. J Am Soc Echocardiogr. 2015;28(1):1-39. e14.

20. Rudski LG, Lai WW, Afilalo J, Hua L, Handschumacher MD, Chandrasekaran K, Solomon SD, Louie EK, Schiller NB. Guidelines for the echocardiographic assessment of the right heart in adults: a report from the American Society of Echocardiography endorsed by the European Association of Echocardiography, a registered branch of the European Society of Cardiology, and the Canadian Society of Echocardiography. J Am Soc Echocardiogr. 2010;23(7):685-713. quiz 786-688.

21. Nagueh SF, Appleton CP, Gillebert TC, Marino PN, Oh JK, Smiseth OA, Waggoner AD, Flachskampf FA, Pellikka PA, Evangelista A. Recommendations for the evaluation of left ventricular diastolic function by echocardiography. J Am Soc Echocardiogr. 2009;22(2):107-33.

22. Anjos LA. Body mass index (body mass.body height-2) as indicator of nutritional status in adults: review of the literature. Rev Saude Publica. 1992;26(6):431-6.

23. Lohman TG, Roche AF, Martorell R. Anthropometric standardization reference manual. Illinois: Human Kinetics; 1988.

24. Jackson AS, Pollock ML, Ward A. Generalized equations for predicting body density of women. Med Sci Sports Exerc. 1980;12(3):175-81.

25. Jackson AS, Pollock ML. Generalized equations for predicting body density of men. Br J Nutr. 1978;40(3):497-504.

26. Caruso P, Albuquerque AL, Santana PV, Cardenas LZ, Ferreira JG, Prina E, Trevizan PF, Pereira MC, lamonti V, Pletsch $R$, et al. Diagnostic methods to assess inspiratory and expiratory muscle strength. J Bras Pneumol. 2015;41(2):110-23.

27. American Thoracic Society/European Respiratory Society. ATS/ERS Statement on respiratory muscle testing. Am J Respir Crit Care Med. 2002;166(4):518-624.

28. Rousseau P, Mahe G, Haj-Yassin F, Durand S, Humeau A, Leftheriotis G, Abraham P. Increasing the "region of interest" and "time of interest", both reduce the variability of blood flow measurements using laser speckle contrast imaging. Microvasc Res. 2011;82(1):88-91.

29. Cordovil I, Huguenin G, Rosa G, Bello A, Kohler O, de Moraes R, Tibirica E. Evaluation of systemic microvascular endothelial function using laser speckle contrast imaging. Microvasc Res. 2012;83(3):376-9.

30. Nascimento BR, Lima MM, Nunes Mdo C, Alencar MC, Costa HS, Pinto Filho MM, Cota VE, Rocha MO, Ribeiro AL. Effects of exercise training on heart rate variability in Chagas heart disease. Arq Bras Cardiol. 2014;103(3):201-8.

31. Amaral Da Silva Souza MV, Santos Soares CC, Rega De Oliveira J, Rosa De Oliveira C, Hargreaves Fialho P, Cunha DM, Cunha DM, Kopiler DA, Rangel Tura B, Batista Da Cunha A. Heart rate variability: analysis of time-domain indices in patients with chronic Chagas disease before and after an exercise program. Rev Port Cardiol. 2013;32(3):219-27.

32. Dreifus LS, Agarwal JB, Botvinick EH, Ferdinand KC, Fisch C, Fisher JD, Kennedy JW, Kerber RE, Lambert CR, Okike ON, et al. Heart rate variability for risk stratification of life-threatening arrhythmias. American College of Cardiology Cardiovascular Technology Assessment Committee. J Am Coll Cardiol. 1993;22(3):948-50.

33. Ribeiro AL, Ferreira LM, Oliveira E, Cruzeiro PC, Torres RM, Rocha MO. Active orthostatic stress and respiratory sinus arrhythmia in patients with Chagas' disease with preserved left ventricular global systolic function. Arq Bras Cardiol. 2004;83(1):40-4. 35-49.

34. Friedwald WT, Levy RI, Fredrickson DS. Estimation of the concentration of low-density lipoprotein cholesterol in plasma, without use of preparative ultracentrifuge. Clin Clem. 1978;18:499-502.

35. Levine LR, Williams JA, Stadtman ER, Shacter E. Carbonyl assays for determination of oxidatively modified proteins. Methods Enzymol. 1994;233:346-57.

36. Ciconelli RM, Ferraz MB, Santos W, Meinão I, Quaresma MR. Brazilian Portuguese version of the SF-36. A reliable and valid quality of life outcome measure. Rev Bras Reumatol. 1999;39(3):143-50.

37. Carvalho VO, Guimaraes GV, Carrara D, Bacal F, Bocchi EA. Validation of the Portuguese version of the Minnesota Living with Heart Failure Questionnaire. Arq Bras Cardiol. 2009;93(1):39-44.

38. Freyssin C, Verkindt C, Prieur F, Benaich P, Maunier S, Blanc P. Cardiac rehabilitation in chronic heart failure: effect of an 8-week, high-intensity interval training versus continuous training. Arch Phys Med Rehabil. 2012;93(8):1359-64.

39. Pinto Dias JC. Human Chagas disease and migration in the context of globalization: some particular aspects. J Trop Med. 2013;2013:789758.

40. Klein N, Hurwitz I, Durvasula R. Globalization of Chagas disease: a growing concern in nonendemic countries. Epidemiol Res Int. 2012;2012(2012):1-13.

41. Bocchi EA, Braga FG, Ferreira SM, Rohde LE, Oliveira WA, Almeida DR, Moreira Mda C, Bestetti RB, Bordignon S, Azevedo C, et al. III Brazilian Guidelines on Chronic Heart Failure. Arq Bras Cardiol. 2009;93(1 Suppl 1):3-70.

42. Cardoso J, Novaes M, Ochiai M, Regina K, Morgado P, Munhoz R, Brancalhao E, Lima M, Barretto AC. Chagas cardiomyopathy: prognosis 
in clinical and hemodynamic profile C. Arq Bras Cardiol. 2010;95(4): 518-23.

43. Rassi S, Barretto AC, Porto CC, Pereira CR, Calaca BW, Rassi DC. Survival and prognostic factors in systolic heart failure with recent symptom onset. Arq Bras Cardiol. 2005;84(4):309-13.

44. Piepoli MF, Corra U, Benzer W, Bjarnason-Wehrens B, Dendale P, Gaita D, McGee $H$, Mendes M, Niebauer J, Zwisler AD, et al. Secondary prevention through cardiac rehabilitation: from knowledge to implementation. A position paper from the Cardiac Rehabilitation Section of the European Association of Cardiovascular Prevention and Rehabilitation. Eur J Cardiovasc Prev Rehabil. 2010:17(1):1-17.

45. Haskell WL, Lee IM, Pate RR, Powell KE, Blair SN, Franklin BA, Macera CA, Heath GW, Thompson PD, Bauman A, et al. Physical activity and public health: updated recommendation for adults from the American College of Sports Medicine and the American Heart Association. Circulation. 2007;116(9):1081-93.

46. Keating SM, Deng X, Fernandes F, Cunha-Neto E, Ribeiro AL, Adesina B, Beyer Al, Contestable P, Custer B, Busch MP, et al. Inflammatory and cardiac biomarkers are differentially expressed in clinical stages of Chagas disease. Int J Cardiol. 2015;199:451-9.

47. Milani RV, Lavie CJ, Mehra MR. Reduction in C-reactive protein through cardiac rehabilitation and exercise training. J Am Coll Cardiol. 2004;43(6):1056-61.

48. Larsen Al, Gjesdal K, Hall C, Aukrust P, Aarsland T, Dickstein K. Effect of exercise training in patients with heart failure: a pilot study on autonomic balance assessed by heart rate variability. Eur J Cardiovasc Prev Rehabil. 2004;11(2):162-7.

49. Zheng H, Luo M, Shen Y, Ma Y, Kang W. Effects of 6 months exercise training on ventricular remodelling and autonomic tone in patients with acute myocardial infarction and percutaneous coronary intervention. J Rehabil Med. 2008;40(9):776-9.

50. Tabet JY, Meurin P, Driss AB, Weber H, Renaud N, Grosdemouge A, Beauvais F, Cohen-Solal A. Benefits of exercise training in chronic heart failure. Arch Cardiovasc Dis. 2009:102(10):721-30

51. Gielen S, Laughlin MH, O'Conner C, Duncker DJ. Exercise training in patients with heart disease: review of beneficial effects and clinical recommendations. Prog Cardiovasc Dis. 2015;57(4):347-55.

\section{Submit your next manuscript to BioMed Central and we will help you at every step:}

- We accept pre-submission inquiries

- Our selector tool helps you to find the most relevant journal

- We provide round the clock customer support

- Convenient online submission

- Thorough peer review

- Inclusion in PubMed and all major indexing services

- Maximum visibility for your research

Submit your manuscript at www biomedcentral.com/submit

) Biomed Central 